\title{
Impact on Inter-Cell Interference of Reference Signal for Interference Rejection Combining Receiver in LTE-Advanced Downlink
}

\author{
Yousuke SANO $^{\dagger \text { a) }}$, Yusuke OHWATARI ${ }^{\dagger}$, Nobuhiko MIKI ${ }^{\dagger}$, Yuta SAGAE $^{\dagger}$, Members , \\ Yukihiko OKUMURA ${ }^{\dagger}$, Senior Member, Yasutaka OGAWA ${ }^{\dagger \dagger}$, Fellow, Takeo OHGANE ${ }^{\dagger \dagger}$, \\ and Toshihiko NISHIMURA ${ }^{\dagger \dagger}$, Members
}

\begin{abstract}
SUMMARY This paper investigates the dominant impact on the interference rejection combining (IRC) receiver due to the downlink reference signal (RS) based covariance matrix estimation scheme. When the transmission modes using the cell-specific RS (CRS) in LTE/LTE-Advanced are assumed, the property of the non-precoded CRS is different from that of the data signals. This difference poses two problems to the IRC receiver. First, it results in different levels of accuracy for the RS based covariance matrix estimation. Second, assuming the case where the CRS from the interfering cell collides with the desired data signals of the serving cell, the IRC receiver cannot perfectly suppress this CRS interference. The results of simulations assuming two transmitter and receiver antenna branches show that the impact of the CRS-to-CRS collision among cells is greater than that for the CRS interference on the desired data signals especially in closed-loop multiple-input multiple-output (MIMO) systems, from the viewpoint of the output signal-to-interference-plus-noise power ratio (SINR). However, the IRC receiver improves the user throughput by more than $20 \%$ compared to the conventional maximal ratio combining (MRC) receiver under the simulation assumptions made in this paper even when the CRS-to-CRS collision is assumed. Furthermore, the results verify the observations made in regard to the impact of inter-cell interference of the CRS for various average received signal-to-noise power ratio (SNR) and signal-to-interference power ratio (SIR) environments.

key words: LTE-Advanced, interference rejection combining, inter-cell interference, reference signal, turbo equalizer
\end{abstract}

\section{Introduction}

The Long-Term Evolution (LTE) was finalized as Release 8 (simply LTE hereafter) by the 3rd Generation Partnership Project (3GPP) [1]. In Japan, NTT DOCOMO launched a commercial LTE service in December 2010 under the new service brand of "Xi" (crossy) [2]. One key feature for LTE is multi-antenna techniques, i.e., multiple-input multiple-output (MIMO) techniques. In LTE-Advanced, to satisfy high-level requirements for the peak and average spectrum efficiency and cell edge user throughput [3], advanced multi-antenna transmission techniques such as higher-order single-user (SU)-MIMO, multi-user (MU)MIMO, and coordinated multi-point (CoMP) transmission/

\footnotetext{
Manuscript received April 24, 2012.

Manuscript revised August 6, 2012.

†The authors are with NTT DOCOMO, INC., Yokosuka-shi, 239-8536 Japan.

${ }^{\dagger \dagger}$ The authors are with the Graduate School of Information Science and Technology, Hokkaido University, Sapporo-shi, 0600814 Japan.

a) E-mail: yousuke.sano.sp@nttdocomo.com

DOI: $10.1587 /$ transcom.E95.B.3728
}

reception were investigated [4]. The interference rejection combining (IRC) receiver [5] was also evaluated [4]. Regarding this IRC receiver, the study item (SI) phase in which fundamental studies were conducted on elemental technologies for LTE-Advanced (Release 11) was completed and a summary of the technical report was presented [6]. Currently, the work item (WI) specification of the IRC receiver is underway [7].

An important goal for LTE-Advanced is to improve the cell edge user throughput while achieving high-speed and high-capacity communications. Toward this goal, two approaches are considered: transmission processing such as CoMP transmission technique and reception processing. In this paper, we focus on the latter approach. To improve the cell edge user throughput, reception processing should suppress the inter-cell interference signals in addition to detecting the desired signal since the cell edge user throughput is severely deteriorated by inter-cell interference. The IRC receiver, which can suppress the inter-cell interference with the aid of multiple receiver antenna branches, is considered to be a promising approach. To suppress the interference signals with a feasible level of complexity, linear filtering based on the minimum mean square error (MMSE) criterion is investigated for the IRC receiver in [6]. This filtering can be extended to iterative signal detection such as a turbo equalizer using soft interference cancellation [8], [9], which can possibly improve the detection performance. Regarding such signal processing based on an iterative manner, the enhancement of the detection performance in the first stage can possibly bring about improvement in the final attainable performance in the last stage. Therefore, this paper focuses on linear filtering based on the MMSE criteria, as a signal processing technique for the first stage. Hereafter, this type of receiver is simply referred to as the "IRC receiver."

The IRC receiver requires knowledge of the interference signals, i.e., the covariance matrix including the interference signals, in addition to knowledge of the desired signal, i.e., the channel matrix of the serving cell. Therefore, to achieve a gain from the IRC receiver, highly accurate estimation schemes of these matrices are important. In practical applications such as LTE/LTE-Advanced, the channel matrix of the serving cell can be estimated using downlink reference signals (RSs). For covariance matrix estimation, 
the RS based scheme proposed in [10] is effective as an accurate estimation as described in [10] and [11]. This scheme separates the serving cell part and the interference and noise part from the covariance matrix. The former part, i.e., the covariance matrix of the serving cell, can be obtained using the channel amplitudes and phases of the serving cell, which are estimated using the RS of the serving cell. In contrast, the latter part, i.e., the covariance matrix including only the interference and noise, can be estimated by subtracting the replica symbols of the serving cell based on the estimated channel matrix and the known RS sequence from the received RS signals. In particular, the estimation accuracy of the latter is more sensitive to the suppression of the interference signals than the former.

In LTE/LTE-Advanced, various transmission modes are supported. To achieve this, multiple RSs, e.g., the cellspecific RS (CRS) and demodulation RS (DM-RS) [12], are defined. In this paper, we focus on the transmission modes using the CRS since these modes are generally used for up to two transmitter antenna branches. The properties of the CRS are different from those of the data signals, especially when precoding transmission is assumed for the data signals, since the CRS is transmitted without precoding. This difference between the CRS and the data signals poses two problems to the IRC receiver. First, it results in different levels of accuracy for the covariance matrix estimation. More precisely, although the estimated covariance matrix including the interference and noise is accurate when the data signals from the interfering cell interfere with the CRS of the serving cell, it is inaccurate when the CRS from the interfering cell collides with the CRS of the serving cell as illustrated in Fig. 1. This is because the covariance matrix including the interference and noise should include the properties of the data signals from the interfering cell in order for the IRC receiver to suppress the interference of the data signals. Second, assuming the case where the CRS from the interfering cell collides with the desired data signals of the serving cell as illustrated in Fig. 1, the effect of suppressing interference signals for the IRC receiver is expected to degrade compared to the case where the data signals interfere among cells. This is because the IRC receiver suppresses the interference data signals from the interfering cells. Therefore, this paper investigates these two factors that degrade the performance of

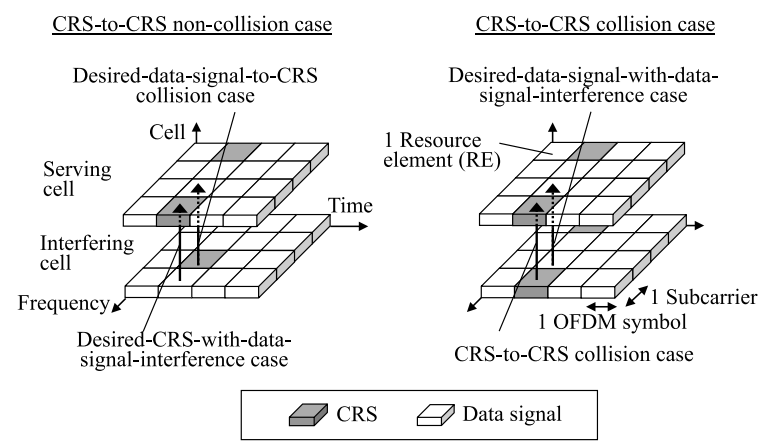

Fig. 1 Inter-cell CRS interference. the IRC receiver.

As the CRS based transmission modes in LTE/LTEAdvanced, closed-loop and open-loop MIMO multiplexing including transmit diversity, i.e., single-stream transmission, are mainly supported. For the closed-loop MIMO system, single-stream precoding transmission is employed based on the Rel. 8 codebook [12]. For the open-loop MIMO system, space-frequency block code (SFBC) using Alamouti coding [13] or large delay cyclic delay diversity (CDD) transmission is employed according to the number of transmission streams, i.e., transmit diversity or MIMO multiplexing [12]. In this paper, both of these transmission modes are evaluated in order to clarify the impact due to the CRS-to-CRS collision among the serving and interfering cells. Note that only transmit diversity is employed in the serving cell since the IRC receiver can improve the cell edge user throughput, and the transmit diversity is mainly used at the cell edge due to the low received signal-to-interference-plus-noise power ratio (SINR). We conduct a multi-cell link level simulation that can be set to an arbitrary number of interfering cells, and evaluate the performance for the IRC receiver from the viewpoints of the output SINR after IRC reception and the user throughput.

In the rest of the paper, Sect. 2 describes the CRS and data signal transmission model. Section 3 describes the IRC receiver weight matrix generation scheme and discusses the impact for CRS-to-CRS collision or desired-data-signal-toCRS collision among cells. Then, the simulation configuration is given in Sect.4. Finally, we present the simulation results in Sect. 5, and our concluding statements in Sect. 6.

\section{CRS and Data Signal Transmission Model}

In this paper, we investigate a case involving the number of transmitter antenna branches, $N_{\mathrm{Tx}}$, of two, which achieves the maximum diversity gain when SFBC using Alamouti coding is employed. Furthermore, in this section, a signal model is described focusing on the IRC receiver assuming the closed-loop MIMO system employing a precoding transmission. Regarding the open-loop transmit diversity employing SFBC, details of the signal model are described in [14].

\subsection{CRS Configuration in LTE/LTE-Advanced}

Figure 2 illustrates the transmit frame structures assuming

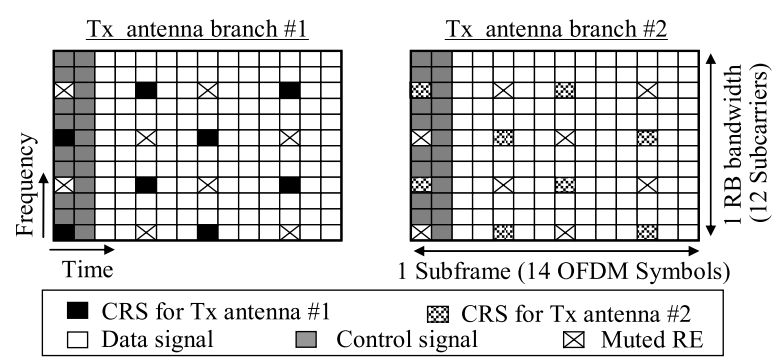

Fig. 2 Transmit frame structure. 
two transmitter antenna branches. One resource block (RB) is shown in the figure, which is the minimum assignment unit defined as 12 subcarriers $\times 14$ OFDM symbols (one subframe). In this paper, a synchronous network where the transmission timing of each subframe is aligned in all cells is assumed. The first two OFDM symbols are assumed to be used for control signaling and the CRS. The CRS is assumed to be multiplexed with the insertion density of 16 resource elements (REs) per RB and transmitted independently from each transmitter antenna branch.

The CRS is transmitted by shifting one subcarrier according to the cell ID; therefore, assuming a synchronous network, the CRSs transmitted from the interfering cells collide with those transmitted from the serving cell every three cells as illustrated in Fig. 1. In contrast, when there is no collision of CRSs among the three cells including the serving and dominant interfering cells, the CRSs transmitted from the interfering cells always interfere with the desired data signals from the serving cell. In the next section, the signal models for each case in addition to more general cases, i.e., the desired-data-signal-with-datasignal-interference and the desired-CRS-with-data-signalinterference cases, are expressed assuming a multi-cell environment.

\subsection{Signal Model in Multi-Cell Environment}

(1) For desired-data-signal-with-data-signal-interference

As a general case, the case where the interfering data signal interferes with the desired data signal of the serving cell is assumed here. Assuming the number of receiver antenna branches as $N_{\mathrm{Rx}}$, the $N_{\mathrm{Rx}}$-dimensional received signal vector of the $k$-th subcarrier and the $l$-th OFDM symbol, $\boldsymbol{y}_{\text {DtoD }}(k, l)$, is expressed as follows.

$$
\boldsymbol{y}_{\mathrm{DtoD}}(k, l)=\sum_{i=0}^{N_{\mathrm{Cell}}-1} \boldsymbol{H}_{i}(k, l) \boldsymbol{W}_{\mathrm{Tx}, i}(k, l) \boldsymbol{s}_{i}(k, l)+\boldsymbol{n}(k, l),
$$

where $\boldsymbol{H}_{i}(k, l)$ represents the $\left(N_{\mathrm{Rx}} \times N_{\mathrm{Tx}}\right)$ channel matrix between the $i$-th cell and the set of user equipment (UE), $\boldsymbol{W}_{\mathrm{Tx}, i}(k, l)$ represents the $\left(N_{\mathrm{Tx}} \times N_{\mathrm{Stream}}\right)$ precoding weight matrix of the $i$-th cell, $s_{i}(k, l)$ represents the $N_{\text {Stream }}{ }^{-}$ dimensional information signal vector of the $i$-th cell, and $\boldsymbol{n}(k, l)$ is the $N_{\mathrm{Rx}}$-dimensional noise vector. Here, $N_{\text {Stream }}$ and $N_{\text {Cell }}$ are the numbers of transmission streams for the UE, i.e., transmission ranks, and the total number of cells, respectively. The 0 -th cell $(i=0)$ is defined as the serving cell for the UE. As mentioned above, $N_{\mathrm{Tx}}$ is assumed to be two in the paper. In the paper, the total transmit data signal power per symbol, $P_{\text {Data }}$, is defined as $P_{\text {Data }}=E\left[\left\|s_{i}(k, l)\right\|^{2}\right]$. Here, $E[\cdot]$ denotes the expectation operation. Note that $\boldsymbol{W}_{\mathrm{Tx}, i}(k, l)$ is the unitary matrix, i.e., $\boldsymbol{W}_{\mathrm{Tx}, i}(k, l) \boldsymbol{W}_{\mathrm{Tx}, i}^{H}(k, l)=\boldsymbol{I}$ where $\boldsymbol{I}$ is the identity matrix, when the open-loop MIMO multiplexing employing large delay CDD in LTE/LTE-Advanced [14] with two transmitter antenna branches is assumed. Furthermore, when open-loop transmit diversity employing SFBC is assumed, $\boldsymbol{W}_{\mathrm{Tx}, i}(k, l)$ is the identity matrix and the channel matrix, $\boldsymbol{H}_{i}(k, l)$, is expressed including the SFBC [14].

(2) For desired-CRS-with-data-signal-interference

In regard to the case where the interfering data signal interferes with the received CRS of the serving cell, the received signal vector, $\boldsymbol{y}_{\mathrm{CtoD}}(k, l)$, is expressed as follows.

$$
\begin{aligned}
& \boldsymbol{y}_{\mathrm{CtoD}}(k, l)=\boldsymbol{H}_{0}(k, l) \boldsymbol{d}_{0}(k, l) \\
& \quad+\sum_{i=1}^{N_{\mathrm{Cell}}-1} \boldsymbol{H}_{i}(k, l) \boldsymbol{W}_{\mathrm{Tx}, i}(k, l) \boldsymbol{s}_{i}(k, l)+\boldsymbol{n}(k, l) \quad\left(k, l \in \boldsymbol{M}_{\mathrm{CRS}}\right),
\end{aligned}
$$

where $\boldsymbol{M}_{\mathrm{CRS}}$ is the CRS RE group in the serving cell. When the $N_{\mathrm{Rx}}$-dimensional channel vector regarding the $m$ th transmitter antenna branch is estimated based on the CRS, the following 2-dimensional CRS sequence vector, $\boldsymbol{d}_{0}(k, l)$, is used as the transmitted signals.

$$
\boldsymbol{d}_{0}(k, l)=\left\{\begin{array}{l}
{\left[\sqrt{P_{\mathrm{CRS}}} d_{1,0}(k, l), 0\right]^{T} \text { for Tx \#1 }} \\
{\left[0, \sqrt{P_{\mathrm{CRS}}} d_{2,0}(k, l)\right]^{T} \text { for Tx \#2 }}
\end{array},\right.
$$

where $P_{\mathrm{CRS}}$ is the transmit power of the CRS and $d_{m, i}(k, l)$ is the CRS sequence at the $m$-th transmitter antenna branch of the $i$-th cell. Here, $P_{\mathrm{CRS}}$, is defined as $P_{\mathrm{CRS}}=E\left[\left\|\boldsymbol{d}_{0}(k, l)\right\|^{2}\right]$. Superscript ${ }^{T}$ denotes the transpose.

\section{(3) For CRS-to-CRS collision}

As mentioned earlier, CRS-to-CRS collision occurs between the serving and interfering cells. When the CRS of the interfering cells, e.g., $i=1$, is assumed to collide with one of the serving cells, the received signal vector, $\boldsymbol{y}_{\mathrm{CtoC}}(k, l)$, is expressed as follows.

$$
\begin{aligned}
& \boldsymbol{y}_{\mathrm{CtoC}}(k, l)=\boldsymbol{H}_{0}(k, l) \boldsymbol{d}_{0}(k, l)+\boldsymbol{H}_{1}(k, l) \boldsymbol{d}_{1}(k, l) \\
& \quad+\sum_{i=2}^{N_{\mathrm{Cell}}-1} \boldsymbol{H}_{i}(k, l) \boldsymbol{W}_{\mathrm{Tx}, i}(k, l) \boldsymbol{s}_{i}(k, l)+\boldsymbol{n}(k, l) \quad\left(k, l \in \boldsymbol{M}_{\mathrm{CRS}}\right) .
\end{aligned}
$$

(4) For desired-data-signal-to-CRS collision

For the last case, it is considered that the CRS of the interfering cell, e.g., $i=1$, collides with the data signal of the serving cell. The received signal vector, $\boldsymbol{y}_{\mathrm{DtoC}}(k, l)$, is expressed as follows.

$$
\begin{aligned}
\boldsymbol{y}_{\mathrm{DtoC}}(k, l)= & \sum_{i=0, i \neq 1}^{N_{\mathrm{Cell}}-1} \boldsymbol{H}_{i}(k, l) \boldsymbol{W}_{\mathrm{Tx}, i}(k, l) \boldsymbol{s}_{i}(k, l) \\
& +\boldsymbol{H}_{1}(k, l) \boldsymbol{d}_{1}(k, l)+\boldsymbol{n}(k, l) .
\end{aligned}
$$

\section{IRC Receiver Weight Matrix Using Estimated Co- variance Matrix}

In this section, an IRC receiver weight matrix generation scheme is described focusing on the closed-loop MIMO system. Regarding open-loop transmit diversity employing the SFBC, the IRC receiver should be considered in terms of the space and code domains [15]. Details of the receiver weight matrix generation scheme are described in [14]. 


\subsection{IRC Receiver Weight Matrix Generation}

The recovered signal vector of the data signals at the UE, $\hat{\boldsymbol{s}}_{0}(k, l)$, is detected by multiplying the $\left(N_{\mathrm{Stream}} \times N_{\mathrm{Rx}}\right)$ receiver weight matrix, $\boldsymbol{W}_{\mathrm{Rx}, 0}(k, l)$, to the received signal vector, $\boldsymbol{y}_{\mathrm{DtoD}}(k, l)$ or $\boldsymbol{y}_{\mathrm{DtoC}}(k, l)$, in Eq. (1) or Eq. (5) as follows.

$$
\hat{\boldsymbol{s}}_{0}(k, l)=\left\{\begin{array}{l}
\boldsymbol{W}_{\mathrm{Rx}, 0}(k, l) \boldsymbol{y}_{\mathrm{DtoD}}(k, l) \\
\boldsymbol{W}_{\mathrm{Rx}, 0}(k, l) \boldsymbol{y}_{\mathrm{DtoC}}(k, l)
\end{array} .\right.
$$

To obtain the IRC receiver weight matrix, the RS based covariance matrix estimation scheme [10] is employed. As the RS, the CRS is assumed in this paper, as described in Sect.1. Using the CRS sequence of the serving cell, which is known at the receiver, the covariance matrix including only the interference and noise component, $\boldsymbol{R}_{I+N}$, is estimated using the received CRS vector, $\boldsymbol{y}_{\mathrm{CtoD}}(k, l)$ or $\boldsymbol{y}_{\text {CtoC }}(k, l)$, in Eq. (2) or Eq. (4) as follows.

$$
\begin{aligned}
\boldsymbol{R}_{I+N} & =\frac{1}{N_{\mathrm{sp}}} \sum_{k, l \in \boldsymbol{M}_{\mathrm{CRS}}} \tilde{\boldsymbol{y}}(k, l) \tilde{\boldsymbol{y}}^{H}(k, l), \\
\tilde{\boldsymbol{y}}(k, l) & =\left\{\begin{array}{l}
\boldsymbol{y}_{\mathrm{CtoD}}(k, l)-\hat{\boldsymbol{H}}_{0}(k, l) \boldsymbol{d}_{0}(k, l) \\
\boldsymbol{y}_{\mathrm{CtoC}}(k, l)-\hat{\boldsymbol{H}}_{0}(k, l) \boldsymbol{d}_{0}(k, l)
\end{array}\left(k, l \in \boldsymbol{M}_{\mathrm{CRS}}\right),\right.
\end{aligned}
$$

where $\hat{\boldsymbol{H}}_{0}(k, l)$ is the estimated channel matrix based on the CRS, $N_{\text {sp }}$ is the number of CRSs for averaging in Eq. (7). Superscript ${ }^{H}$ denotes the Hermitian transpose. Note that the UE is informed of the information of the precoding matrix in the serving cell, $\boldsymbol{W}_{\mathrm{Tx}, 0}(k, l)$, in the CRS based closed-loop MIMO system in LTE/LTE-Advanced. Using the estimated $\boldsymbol{R}_{I+N}$ and $\hat{\boldsymbol{H}}_{0}(k, l)$, the covariance matrix, $\boldsymbol{R}_{\mathrm{yy}}(k, l)$, is estimated using the following equation.

$$
\begin{aligned}
\boldsymbol{R}_{\mathrm{yy}}(k, l)= & \frac{P_{\text {Data }}}{N_{\text {Stream }}} \hat{\boldsymbol{H}}_{0}(k, l) \boldsymbol{W}_{\mathrm{Tx}, 0}(k, l) \boldsymbol{W}_{\mathrm{Tx}, 0}^{H}(k, l) \hat{\boldsymbol{H}}_{0}^{H}(k, l) \\
& +\boldsymbol{R}_{I+N} .
\end{aligned}
$$

Assuming equal power allocation for each data stream, the IRC receiver weight matrix is calculated using $\boldsymbol{R}_{\mathrm{yy}}(k, l)$ as follows.

$$
\boldsymbol{W}_{\mathrm{IRC}}(k, l)=\frac{P_{\text {Data }}}{N_{\text {Stream }}}\left(\hat{\boldsymbol{H}}_{0}(k, l) \boldsymbol{W}_{\mathrm{Tx}, 0}(k, l)\right)^{H} \boldsymbol{R}_{\mathrm{yy}}^{-1}(k, l) .
$$

\subsection{Impact on Estimation Accuracy of Covariance Matrix for CRS-to-CRS Collision}

In this section, we discuss the impact on the estimation accuracy of the covariance matrix for the CRS-to-CRS collision compared to that for the desired-CRS-with-data-signalinterference.

\section{(1) For desired-CRS-with-data-signal-interference}

First, the case where the data signals of the interfering cells interfere with the CRS of the serving cell is assumed. Using the RS based estimation scheme expressed in Eq. (7) and Eq. (8), the covariance matrix that only includes the interference and noise, $\boldsymbol{R}_{I+N}$, for the closed- and open-loop MIMO systems can be estimated in principle by using the received CRS vector, $\boldsymbol{y}_{\mathrm{CtoD}}(k, l)$ in Eq. (2) as follows.

$\left\{\begin{array}{l}\boldsymbol{R}_{I+N} \cong \sum_{i=1}^{N_{\text {Cell }}-1} \frac{P_{\text {Data }}}{N_{\text {Stram }}} H_{i} \boldsymbol{W}_{\mathrm{Tx}, i} \boldsymbol{W}_{\mathrm{T}, i}^{H} \boldsymbol{H}_{i}^{H}+\sigma^{2} \boldsymbol{I} \text { for closed-loop } \\ \boldsymbol{R}_{I+N} \cong \sum_{i=1}^{N_{\text {Cell }}-1} \frac{P_{\text {Daat }}}{2} \boldsymbol{H}_{i} \boldsymbol{H}_{i}^{H}+\sigma^{2} \boldsymbol{I} \text { for open-loop }\end{array}\right.$,

where $\sigma^{2}$ is the noise power. Note that the channel fluctuations in the time and frequency domains are assumed to be negligible within $1 \mathrm{RB}$ and the description of $(k, l)$ is omitted for simplicity. Furthermore, regarding the open-loop MIMO multiplexing and transmit diversity, $\boldsymbol{W}_{\mathrm{Tx}, i} \boldsymbol{W}_{\mathrm{Tx}, i}^{H}=\boldsymbol{I}$ and $\boldsymbol{W}_{\mathrm{Tx}, i}=\boldsymbol{I}$ are considered, respectively. Each data signal power is also assumed to be $P_{\text {Data }} / 2$ for both 1 and 2-stream transmissions.

\section{(2) For CRS-to-CRS collision}

In contrast, the CRS-to-CRS collision among the serving and interfering cells is assumed. Substituting the received CRS vector, $\boldsymbol{y}_{\mathrm{CtoC}}(k, l)$, in Eq. (4) into Eq. (7) and Eq. (8), the estimated covariance matrix that includes only the interference and noise becomes the following expression in principle.

$$
\left\{\begin{array}{l}
\boldsymbol{R}_{I+N} \cong \frac{P_{\mathrm{CRS}}}{2} \boldsymbol{H}_{1} \boldsymbol{H}_{1}^{H} \\
\quad+\sum_{i=2}^{N_{\mathrm{Cell}}-1} \frac{P_{\mathrm{Data}}}{N_{\mathrm{Stream}}} \boldsymbol{H}_{i} \boldsymbol{W}_{\mathrm{T} \mathrm{T}, i} \boldsymbol{W}_{\mathrm{T}, i}^{H} \boldsymbol{H}_{i}^{H}+\sigma^{2} \boldsymbol{I} \text { for closed-loop } \\
\boldsymbol{R}_{I+N} \cong \frac{P_{\mathrm{CRS}}}{2} \boldsymbol{H}_{1} \boldsymbol{H}_{1}^{H}+\sum_{i=2}^{N_{\mathrm{Cell}}-1} \frac{P_{\mathrm{Data}}}{2} \boldsymbol{H}_{i} \boldsymbol{H}_{i}^{H}+\sigma^{2} \boldsymbol{I} \text { for open-loop }
\end{array}\right.
$$

Note that this equation can be formulated when the number of CRSs for each transmitter antenna branch is the same as that illustrated in Fig. 2.

When comparing Eq. (11) and Eq. (12), the property of the estimated covariance matrix is changed due to the CRSto-CRS collision when $\boldsymbol{W}_{\mathrm{Tx}, i}$ is not the unitary matrix, i.e., $\boldsymbol{W}_{\mathrm{Tx}, i} \boldsymbol{W}_{\mathrm{Tx}, i}^{H} \neq \boldsymbol{I}$, for the closed-loop MIMO system. Since a change in the property of the covariance matrix means that the accuracy of the covariance matrix estimation becomes degraded, the performance for the IRC receiver is expected to be degraded in this system. On the other hand, regarding the open-loop MIMO system, no degradation of the performance for the IRC receiver is expected since the property of the estimated covariance matrix is not changed under the assumption $P_{\text {Data }}=P_{\mathrm{CRS}}$.

3.3 Impact on Effect of Suppressing Interference for Desired-Data-Signal-to-CRS Collision

Considering the desired-data-signal-to-CRS collision expressed in Eq. (5), the CRSs transmitted from the interfering cells interfere with the data signals transmitted from the serving cell, i.e., non-precoded interference signals affect the desired data signals. The IRC receiver can suppress the interference of data signals transmitted from the interfering cells. Therefore, especially in the closed-loop MIMO 
system employing precoding, the effect of suppressing the interference for the IRC receiver is expected to degrade due to the different properties of the interference signals, i.e., precoded or non-precoded signals. However, the number of desired data signals that incur interference from the CRSs transmitted from one interfering cell is limited, i.e., only 12 $\mathrm{RE} / \mathrm{RB}$ under the assumptions of the CRS insertion density as illustrated in Fig. 2 and a synchronous network. Therefore, the impact on the IRC receiver due to the inter-cell interference of the CRS is expected to be small compared to that due to the CRS-to-CRS collision.

\section{Simulation Conditions}

To investigate the impact on the IRC receiver due to the CRS interference, the output SINR and the throughput performance for the IRC receiver are evaluated. In this paper, the output SINR and throughput performance levels are evaluated for both CRS-to-CRS collision and CRS-to-CRS noncollision cases as illustrated in Fig. 1. Note that each case includes the following cases and influences as described in Sects. 2.2, 3.2, and 3.3.

- CRS-to-CRS collision case:

Includes both the CRS-to-CRS collision case as in Sect. 2.2 (3) and the desired-data-signal-with-datasignal-interference case as in Sect. 2.2 (1). The former case impacts the estimation accuracy of the covariance matrix as described in Sect. 3.2. The latter case impacts the effect of suppressing interference as described in Sect. 3.3.

- CRS-to-CRS non-collision case:

Includes both the desired-CRS-with-data-signal-interference case as in Sect. 2.2 (2) and the desired-datasignal-to-CRS collision case as in Sect. 2.2 (4). In addition to the CRS-to-CRS collision case, the former case impacts the estimation accuracy of the covariance matrix as described in Sect. 3.2. The latter case impacts the effect of suppressing interference as described in Sect. 3.3.

Here, the number of the interfering cells is assumed to be two, i.e., the three-cell model is assumed. For the CRSto-CRS collision case, the CRSs transmitted from the most dominant interfering cell collide with the CRSs transmitted from the serving cell. In contrast, for the CRS-to-CRS noncollision case, it is assumed that there are no collisions of CRSs among the three cells.

Regarding the output SINR, since we assume that only transmit diversity, i.e., single-stream transmission, is employed in the serving cell, a single output SINR is obtained in this paper. For calculating the output SINR, the following formula is used when the closed-loop MIMO system is assumed.

$$
\operatorname{SINR}=\frac{\boldsymbol{W}_{\mathrm{Rx}, 0} P \boldsymbol{H}_{0} \boldsymbol{W}_{\mathrm{Tx}, 0} \boldsymbol{W}_{\mathrm{Tx}, 0}^{H} \boldsymbol{H}_{0}^{H} \boldsymbol{W}_{\mathrm{Rx}, 0}^{H}}{\boldsymbol{W}_{\mathrm{Rx}, 0}\left(\sum_{i=1}^{N_{\mathrm{cell}}-1} P \boldsymbol{H}_{i} \boldsymbol{W}_{\mathrm{Tx}, i} \boldsymbol{W}_{\mathrm{Tx}, i}^{H} \boldsymbol{H}_{i}^{H}+\sigma^{2} \boldsymbol{I}\right) \boldsymbol{W}_{\mathrm{Rx}, 0}^{H}} .
$$

Here, the description of $(k, l)$ is omitted for simplicity. When the open-loop MIMO multiplexing is assumed, $\boldsymbol{W}_{\mathrm{Tx}, i} \boldsymbol{W}_{\mathrm{Tx}, i}^{H}$ is the identity matrix in this paper due to the assumption of the two transmitter antenna branches. Furthermore, if openloop transmit diversity employs the SFBC, the channel matrix, $\boldsymbol{H}_{i}(k, l)$, is expressed including the SFBC.

For the performance evaluation, a multi-cell link level simulation is conducted. Note that a link level simulation is performed between each UE and its serving cell as well as the neighboring cells in this simulation.

The numbers of transmitter and receiver antenna branches are assumed to be two and two, respectively. Therefore, the spatial degree of freedom is one when transmit diversity is assumed. Fifty consecutive RBs, i.e., $9 \mathrm{MHz}$, are assigned to the UE in the serving cell. However, the assignment granularity of each UE in the interfering cells is assumed to be six consecutive RBs. Furthermore, 50 consecutive RBs are all assigned to 9 UEs in the interfering cells. In the evaluation, although the insertion density of the CRS is $16 \mathrm{RE} / \mathrm{RB}$ as illustrated in Fig. 2, the CRSs located in the data signal region are only used for the RS based covariance matrix estimation in order to exclude the impact of the interference of control signals. This is because a synchronous network is assumed in this paper. The covariance matrix is estimated within $1 \mathrm{RB}$. Therefore, the number of averaging samples, $N_{\mathrm{sp}}$, equals 12 in Eq. (7). The channel estimation scheme for the CRS is assumed to be the 2-dimensional MMSE channel estimation scheme [16]. For the MMSE channel estimation filter, a uniform delay power spectrum within the cyclic prefix length of $4.69 \mu \mathrm{s}$ is assumed in the frequency domain, and a uniform Doppler power spectrum with the maximum Doppler frequency of $5.55 \mathrm{~Hz}$ is assumed in the time domain. The total data signal power per symbol, $P_{\text {Data }}$, is assumed to be same as the CRS power, $P_{\mathrm{CRS}}$.

For the throughput evaluation, hybrid automatic repeat request (ARQ) with incremental redundancy is employed and the maximum number of retransmissions is assumed to be four. Outer-loop link adaptation [17] is employed with the target BLER of 10\%. Furthermore, MIMO multiplexing with two-stream transmission is used in the interfering cells in addition to the transmit diversity for the practical throughput evaluation. The probability of transmit diversity is assumed to be $80 \%$ every $6 \mathrm{RBs}$ assigned to each UE in the interfering cells.

The assumption for the closed-loop MIMO system is that the two transmitter antenna codebooks defined in [12] are used for precoding transmission. Regarding the serving cell, based on the estimated channel matrix using the CRS, the UE selects the precoding weight matrix, which maximizes the received SINR from the codebook, and then the selected precoding weight matrix information at the UE is fed back to the serving cell. In this paper, the precoding granularity is assumed to be the whole bandwidth, i.e., 50 $\mathrm{RBs}$, in the frequency domain and every subframe, i.e., $1 \mathrm{~ms}$, in the time domain. Furthermore, we assume that there is no feedback error. Note that the precoding weight matrix is 
Table 1 Simulation parameters.

\begin{tabular}{|c|c|}
\hline Carrier frequency / System bandwidth & $2 \mathrm{GHz} / 10 \mathrm{MHz}$ \\
\hline Resource allocation & $50 \mathrm{RBs}$ \\
\hline MIMO antenna configuration & $2 \times 2$ (uncorrelated) \\
\hline Channel model & $(357 \mathrm{~ns})$ \\
(r.m.s. delay spread) & $5.55 \mathrm{~Hz}$ \\
\hline Maximum Doppler frequency & QPSK $(R=0.11-0.61)$ \\
\hline Modulation and coding scheme & $16 \mathrm{QAM}(R=0.33-0.54)$ \\
(MCS) & 64QAM $(R=0.39-0.93)$ \\
\hline MCS transmission granularity & Randomly changing every $1 \mathrm{~ms}$ \\
on interfering cells & Frequency granularity: 6 RBs \\
\hline Channel estimation & 2D-MMSE channel estimation \\
\hline Hybrid ARQ (Round trip delay) & Incremental Redundancy (8 ms) \\
\hline Scheduling / Feedback delay & $8 \mathrm{~ms} / 5 \mathrm{~ms}$ \\
\hline
\end{tabular}

randomly selected from the codebook for every subframe in the interfering cells for simplicity. Regarding the open-loop MIMO multiplexing employing the large delay CDD, the precoding weight matrix is defined in [12].

In the evaluation, the performance for not only the IRC receiver but also the conventional maximal ratio combining (MRC) receiver, which is equal to the MMSE receiver assuming transmit diversity, is evaluated for comparison. The other simulation parameters are given in Table 1.

\section{Performance Evaluation}

\subsection{Output SINR Performance Evaluation}

In the output SINR evaluation, transmit diversity is employed at all the cells and the received signal-to-interference power ratios (SIRs) for the interfering cells are assumed to be $0 \mathrm{~dB}$ and $6 \mathrm{~dB}$. Furthermore, the average received signalto-noise power ration (SNR) is assumed to be $10 \mathrm{~dB}$.

\section{(1) CDF of output SINR}

First, to investigate which impact is the dominant degrading factor, i.e., the CRS-to-CRS collision case or the CRS-toCRS non-collision case, the cumulative distribution function (CDF) of the output SINR of each RE within $50 \mathrm{RBs}$ is evaluated.

Figures 3(a) and 3(b) show the CDF results of the output SINR for open- and closed-loop transmit diversity, respectively. Focusing on open-loop transmit diversity in Fig. 3(a), the performance for the IRC receiver is almost the same regardless of the CRS-to-CRS collision and CRS-toCRS non-collision cases. Therefore, we can say that the impacts of the CRS-to-CRS collision and CRS-to-CRS noncollision cases, i.e., the estimation accuracy of the covariance matrix and the effect of suppressing the interference, are negligible when open-loop transmit diversity is assumed.

However, focusing on closed-loop transmit diversity in Fig. 3(b), the performance for the CRS-to-CRS collision case is degraded compared to that for the CRS-to-CRS noncollision case. Therefore, it is clear that the impact on the estimation accuracy of the covariance matrix for the CRSto-CRS collision case is dominant compared to the impact on the effect of suppressing interference for the CRS-toCRS non-collision case when closed-loop transmit diversity

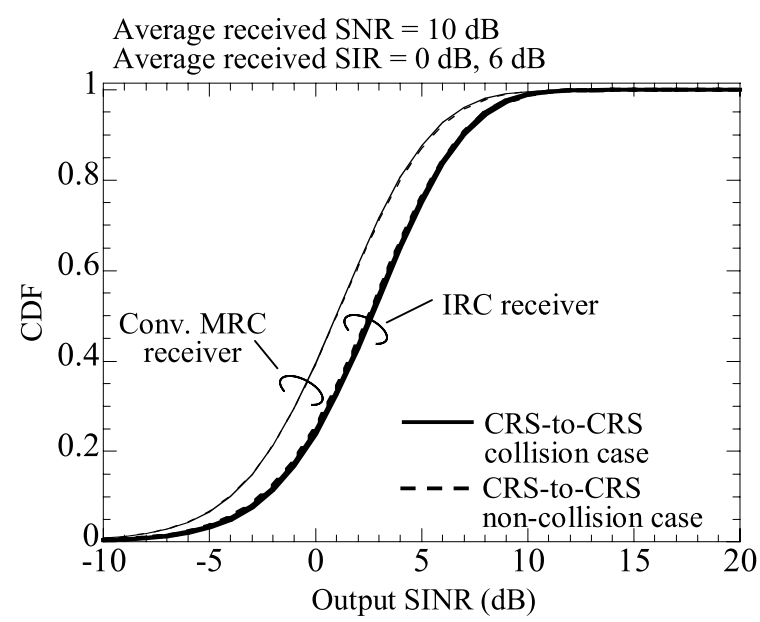

(a) Open-loop transmit diversity using SFBC

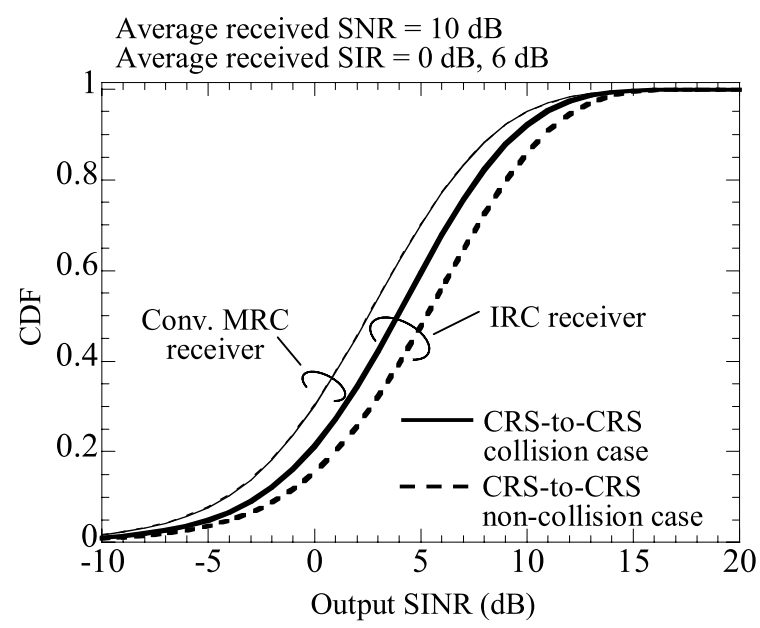

(b) Closed-loop transmit diversity using precoding

Fig. 3 CDF of output SINR.

is assumed.

To investigate the reasons for these phenomena, the average output SINR for each subcarrier is evaluated in the following sections.

(2) Average output SINR for each subcarrier focusing on open-loop transmit diversity

To investigate the reason why the impacts of the CRS-toCRS collision and CRS-to-CRS non-collision cases are negligible for open-loop transmit diversity, the output SINR performance is evaluated focusing on the OFDM symbol where the CRS is not transmitted. This evaluation clarifies the impact of the estimation accuracy of the covariance matrix due to the CRS-to-CRS collision case. Note that when assuming the CRS-to-CRS non-collision case as mentioned in Sect. 4, only the case where the interfering data signal interferes with the desired CRS can be considered.

Figure 4(a) shows the performance results of the average output SINR for each subcarrier within 3 RBs extracted from $50 \mathrm{RBs}$ assuming the open-loop transmit diversity. In this paper, we define the average output SINR per subcar- 


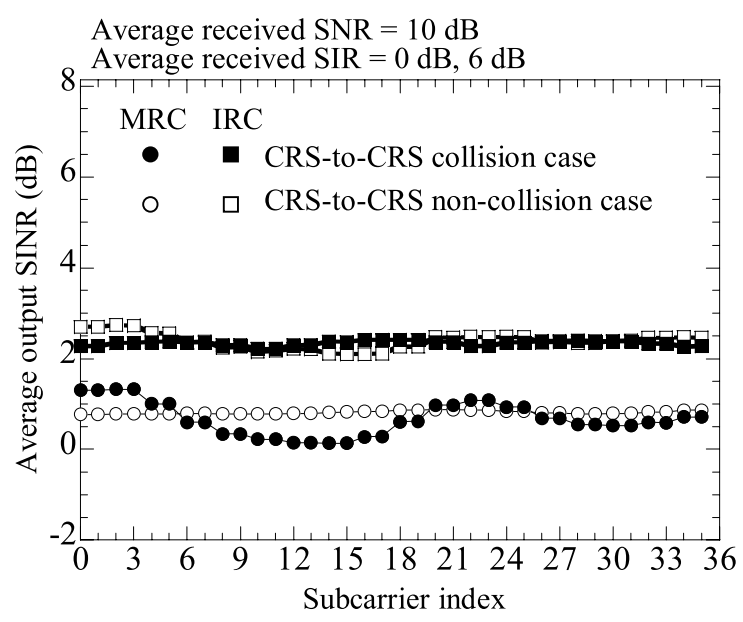

(a) OFDM symbol in CRS non-transmission

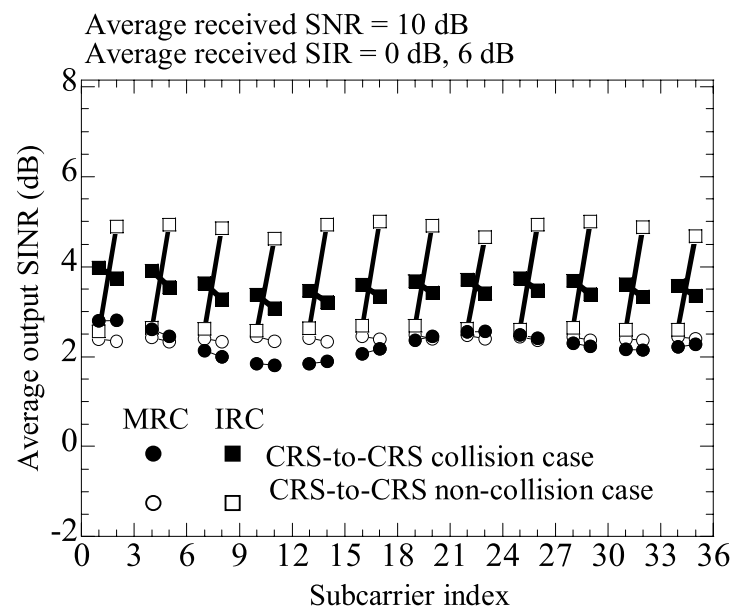

(b) OFDM symbol in CRS transmission

Fig. 4 Average output SINR for each subcarrier (open-loop transmit diversity).

rier on a logarithmic scale for 10,000 subframes in an i.i.d. fading channel. From the results in Fig.4(a), the performance for the IRC receiver is almost the same regardless of the CRS-to-CRS collision and CRS-to-CRS non-collision cases since the property of the estimated covariance matrix, $\boldsymbol{R}_{I+N}$, does not change for either case, which is discussed in Sect. 3.2.

Additionally, to clarify the impact on the effect of suppressing interference for the desired-data-signal-to-CRS collision, i.e., the CRS-to-CRS non-collision case, we evaluate the average output SINR focusing on the OFDM symbol where the CRS is transmitted.

Figure 4(b) shows the performance results of the average output SINR for each subcarrier in the above case. Note that the CRSs are transmitted every three subcarriers; therefore, there are no performance results for the subcarrier indices of $3 q(q \geq 0)$. From the results in Fig. 4(b), the performance for the IRC receiver is almost the same regardless of the CRS-to-CRS collision case, CRS-to-CRS non-collision case, or the previous evaluation results.
Therefore, from these results, we can say that the impacts on the IRC receiver due to the CRS-to-CRS collision and CRS-to-CRS non-collision cases are negligible when assuming the open-loop transmit diversity.

Note that the performance fluctuation for the CRS-toCRS collision case is large compared to that for the CRS-toCRS non-collision case. The reason for this phenomenon is considered to be that the channel estimation accuracy fluctuates based on the CRS from the serving cell since the CRS sequence does not change among subframes, i.e., the CRS from the serving cell suffers from the same CRS interference signal among all subframes.

(3) Average output SINR for each subcarrier focusing on closed-loop transmit diversity

To investigate the reason why the impact of the CRS-toCRS collision is larger than that for the non-CRS-to-CRS collision case for closed-loop transmit diversity, we evaluate the average output SINR considering the same evaluation assumptions described in the previous section except for open-loop transmit diversity.

Figures 5(a) and 5(b) show the performance results of the average output SINR for each subcarrier within 3 RBs extracted from $50 \mathrm{RBs}$ focusing on the OFDM symbol where the CRS is not transmitted and transmitted, respectively.

Focusing on the results in Fig. 5(a), the performance for the CRS-to-CRS collision case is degraded compared to that for the CRS-to-CRS non-collision case. This is because the property of the estimated covariance matrix is changed due to the CRS-to-CRS collision as formulated in Eq. (11) and Eq. (12), i.e., the estimated covariance matrix does not include the precoding weight matrix for the CRS-to-CRS collision case as described in Sect. 3.2. However, when comparing the results for the IRC receiver and MRC receiver, a gain from the IRC receiver can be achieved for all cases. Note that the reason why the performance for the IRC receiver is periodical every RB, i.e., every 12 subcarriers, is that the covariance estimation period is assumed to be $1 \mathrm{RB}$ in this evaluation.

In contrast, focusing on the results in Fig. 5(b), the performance for the IRC receiver assuming the CRS-to-CRS non-collision case is degraded especially for subcarrier indices $(3 q+1)$. This is because the CRS transmitted from the most dominant interfering cell ( $\mathrm{SIR}=0 \mathrm{~dB})$ interferes with the desired data signals. When comparing the performance results for the CRS-to-CRS collision case, the results for subcarrier indices $(3 q+1)$ assuming the CRS-to-CRS noncollision case are severely degraded. Therefore, for only the REs that collide with the CRS from the interfering cell, it is clear that there is a large impact on the effect of suppressing the interference for the CRS-to-CRS non-collision case due to the CRS interference on the desired data signals.

However, based on the previous CDF results of the output SINR in Fig. 3(b), we can say that this impact on the IRC receiver due to the inter-cell interference of the CRS is small compared to that due to the CRS-to-CRS collision, i.e., the 


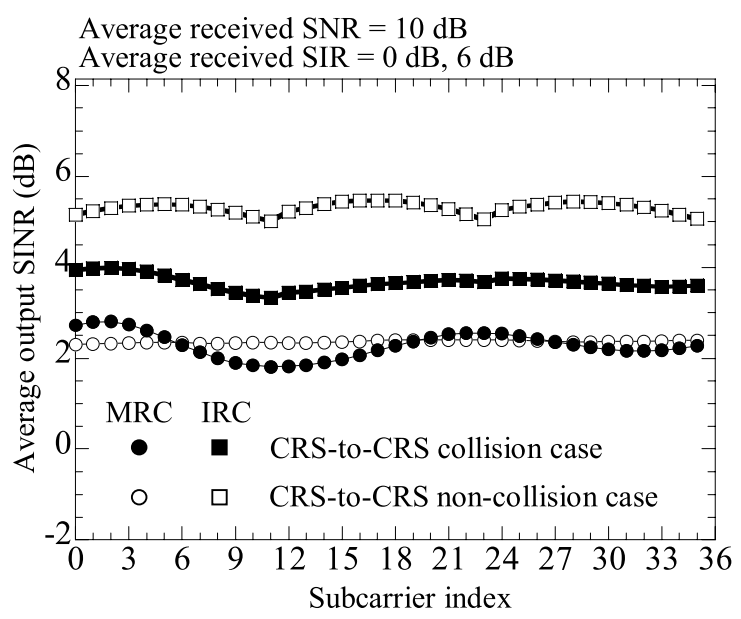

(a) OFDM symbol in CRS non-transmission

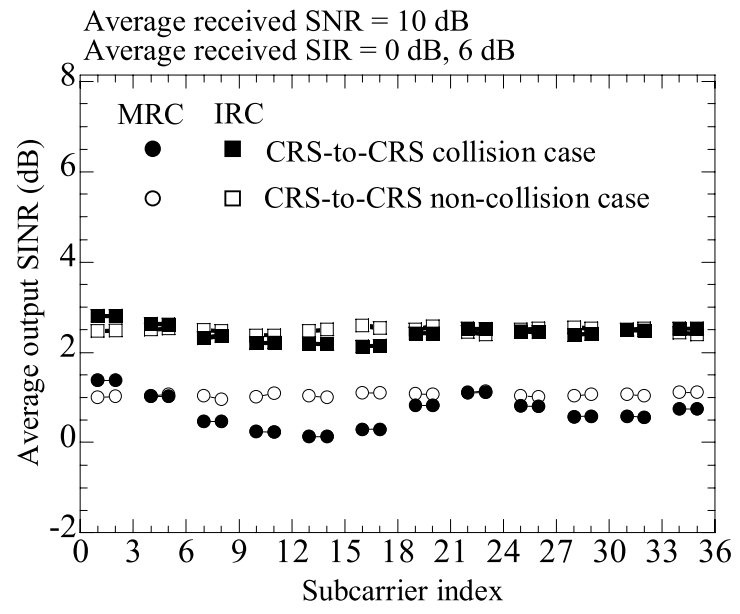

(b) OFDM symbol in CRS transmission

Fig. 5 Average output SINR for each subcarrier (closed-loop transmit diversity).

degradation of the covariance matrix estimation accuracy, when closed-loop transmit diversity is assumed. We consider that this is because the number of desired data signals that incur interference from the CRSs transmitted from one interfering cell is limited, which is expected as described in Sect. 3.3.

\subsection{Throughput Performance Evaluation}

The throughput performance is evaluated in this section. Figures 6(a) and 6(b) show the performance results for the IRC and MRC receiver assuming open- and closed-loop transmit diversity, respectively. Note that the average received SIRs and SNR are assumed to be the same as those for the output SINR evaluation. In regard to the open-loop MIMO system in Fig.6(a), the results show that the gain from the IRC receiver compared to that for the MRC receiver is almost the same regardless of the CRS-to-CRS collision and CRS-to-CRS non-collision cases, which corresponds to the above discussion. Furthermore, regarding the closed-loop MIMO system in Fig. 6(b), the gain from the

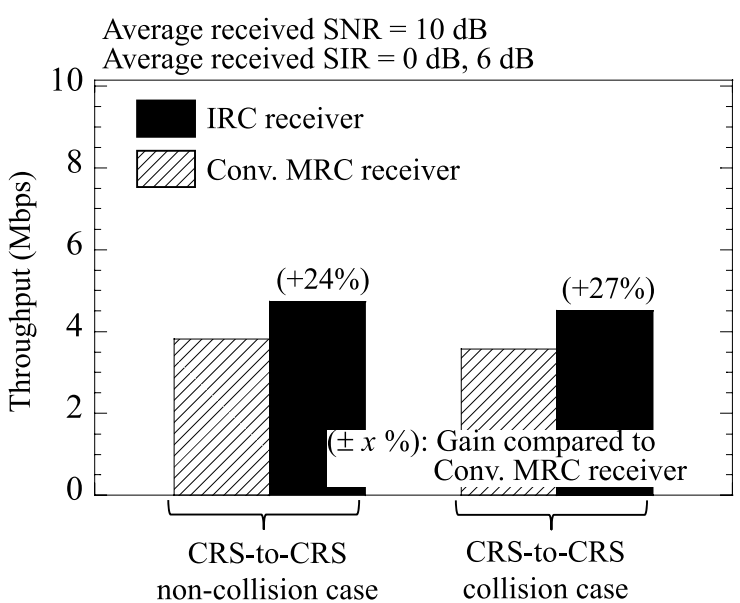

(a) Open-loop transmit diversity using SFBC

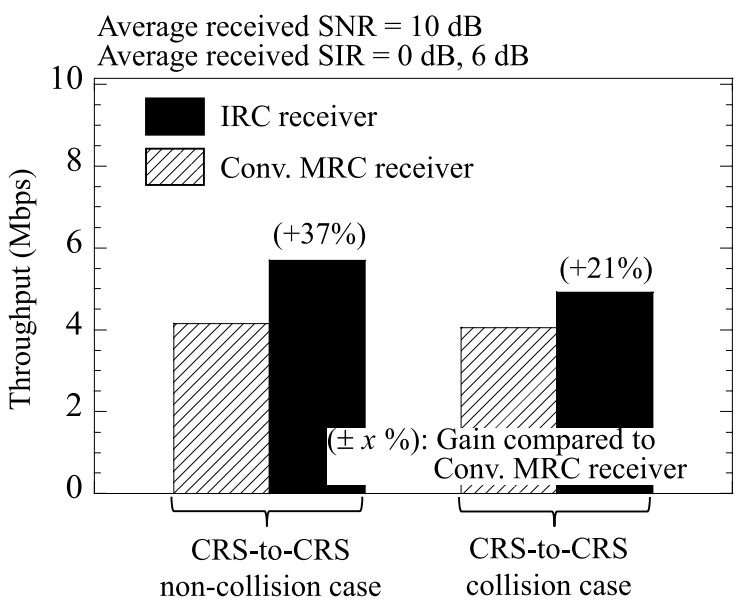

(b) Closed-loop transmit diversity using precoding

Fig. 6 Throughput performance.

IRC receiver in the CRS-to-CRS collision case is degraded compared to the gain in the CRS-to-CRS non-collision case. The gain however, can be achieved even when the CRS-toCRS collision case is assumed.

To confirm the previous observations in various environments, i.e., various average received SNRs and SIRs, we additionally evaluated the throughput performance. In the additional evaluation, the average received SNR is assumed to be from $0 \mathrm{~dB}$ to $30 \mathrm{~dB}$ under the assumption of the average received SIR for each interfering cell of $0 \mathrm{~dB}$ and $6 \mathrm{~dB}$, respectively. Figures 7(a) and 7(b) show the results of the throughput versus the average received SNR for open- and closed-loop transmit diversity, respectively. Focusing on the low SNR region for both results, the gain from the IRC receiver is reduced compared to that in the middle and high SNR regions regardless of the CRS-to-CRS collision case or the CRS-to-CRS non-collision case. This is because the effect of the null beamforming at the IRC receiver is degraded due to the noise-limited environment. However, assuming the case where the average received SNR is over $0 \mathrm{~dB}$, the tendency of the throughput performance corresponds to the previous observations. Specifically, although 


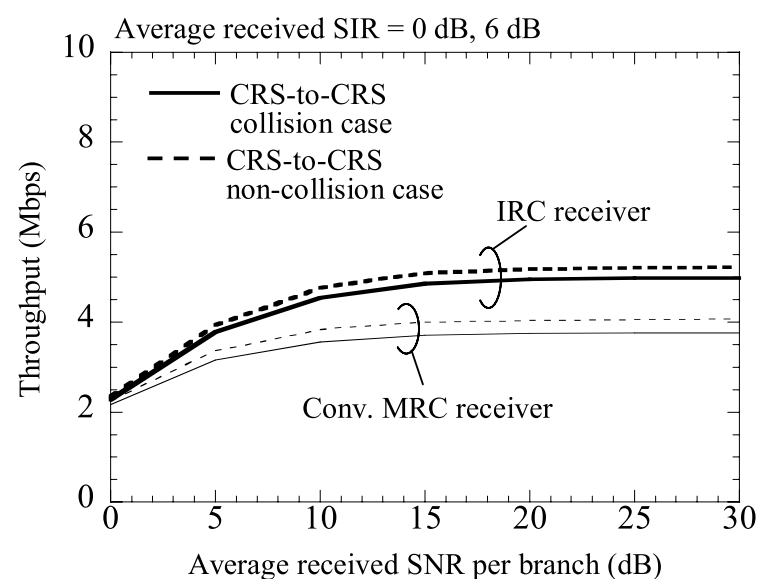

(a) Open-loop transmit diversity using SFBC

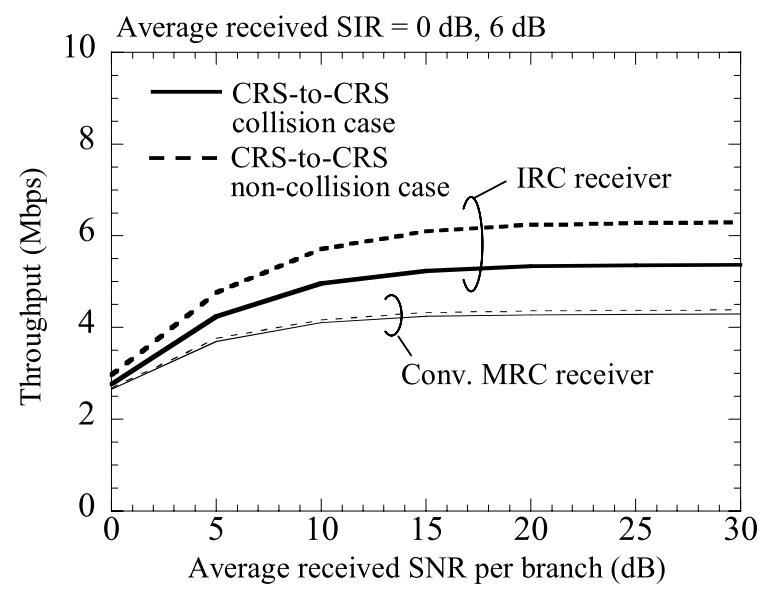

(b) Closed-loop transmit diversity using precoding

Fig. 7 Throughput vs. average received SNR.

the gain from the IRC receiver is almost the same regardless of the CRS-to-CRS collision and CRS-to-CRS non-collision cases assuming open-loop transmit diversity, the gain from the IRC receiver for the CRS-to-CRS collision case is degraded compared to that for the CRS-to-CRS non-collision case assuming closed-loop transmit diversity. Furthermore, we confirmed that a gain is achieved even for the CRS-toCRS collision case when the average received SNR is over $0 \mathrm{~dB}$.

Finally, we evaluated the throughput performance when the average received SIR for the dominant interfering cell is assumed to be from $-3 \mathrm{~dB}$ to $6 \mathrm{~dB}$ under the assumption of the average received SIR for the other interfering cell of $6 \mathrm{~dB}$ and the average received SNR of $10 \mathrm{~dB}$. In this evaluation, the case where the received signal power from the interfering cell is stronger than that of the serving cell is also considered, i.e., SIR of less than $0 \mathrm{~dB}$. This is because a handover margin called a hysteresis is considered in this paper to avoid an excess number of handovers, i.e., a pingpong effect in which the user is handed back and forth several times from one base station to the other when the user moves around the cell boundary. Note that this handover

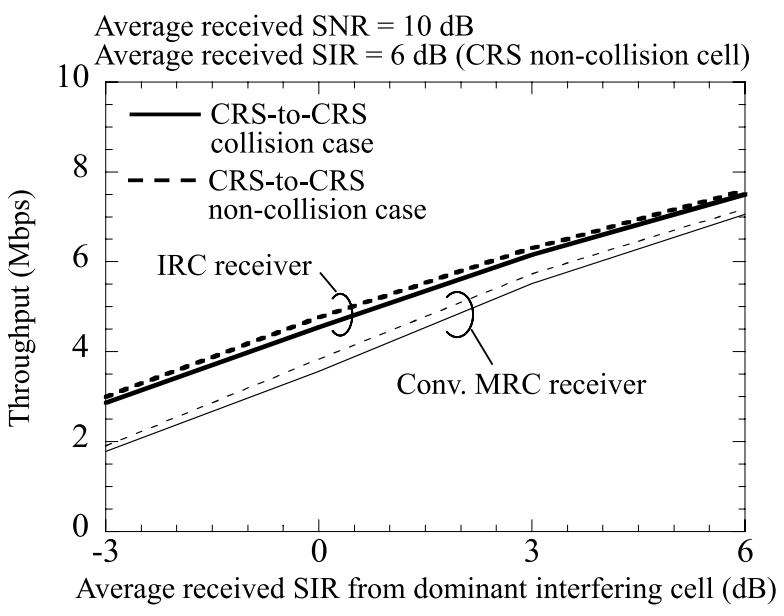

(a) Open-loop transmit diversity using SFBC

Average received SNR $=10 \mathrm{~dB}$

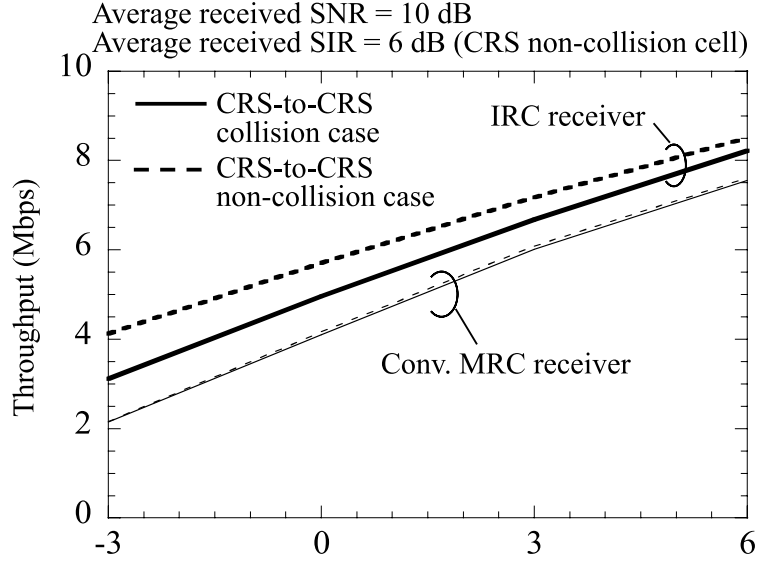

Average received SIR from dominant interfering cell $(\mathrm{dB})$

(b) Closed-loop transmit diversity using precoding

Fig. 8 Throughput vs. average received SIR from dominant interfering cell.

hysteresis is assumed to be set to $3 \mathrm{~dB}$ as an example case in this paper.

Figures 8 (a) and 8 (b) show the results of the throughput versus the average received SIR for the dominant interfering cell, assuming open- and closed-loop transmit diversity, respectively. The results show that the performance tendency also corresponds to the previous observations regardless of the average received SIR for the dominant interfering cell.

Based on all the results, we can say that the gain from the IRC receiver is achieved even for the CRS-to-CRS collision case when the transmitter and receiver antenna branches are assumed to be two and two, respectively, although the impact on the estimation accuracy of the covariance matrix for the CRS-to-CRS collision case is dominant compared to that on the effect of suppressing interference for the CRS-to-CRS non-collision case assuming closed-loop transmit diversity. 


\section{Conclusion}

In this paper, we investigated which factor, i.e., the impact of the CRS-to-CRS collision among cells or the impact of the CRS interference on the desired data signals, was dominant in both closed- and open-loop MIMO multiplexing systems. The results of simulations assuming the LTE/LTE-Advanced downlink with two transmitter and receiver antenna branches showed that the impact of the CRSto-CRS collision among cells was larger than the impact of the CRS interference on the desired data signals especially for the closed-loop system although these impacts did not seriously affect the performance of the open-loop MIMO system, from the viewpoint of the output SINR. However, even when CRS-to-CRS collisions among cells occurred in the closed-loop MIMO system, the IRC receiver could improve the throughput by more than $20 \%$ compared to that for the conventional MRC receiver under the simulation assumptions in this paper. Furthermore, this paper evaluated the throughput performance assuming various average received SNRs and SIRs. The results showed that the observations for the impact of inter-cell interference of the CRS are verified in various environments from the viewpoint of the throughput performance.

\section{References}

[1] 3GPP, TS36.201 (V8.1.0), "LTE physical layer - general description," Nov. 2007.

[2] http://www.nttdocomo.com/pr/2010/001494.html

[3] 3GPP, TR36.913 (V8.0.0), "Requirements for further advancements for Evolved Universal Terrestrial Radio Access (E-UTRA) (LTEAdvanced)," June 2008.

[4] 3GPP, TR36.814 (V1.0.0), "Further advancements for E-UTRA physical layer aspects," Jan. 2009.

[5] J. Winters, "Optimum combining in digital mobile radio with cochannel interference," IEEE J. Sel. Areas Commun., vol.SAC-2, no.4, pp.528-539, April 1984.

[6] 3GPP, TR36.829 (V11.0.0), "Enhanced performance requirement for LTE UE," March 2012.

[7] 3GPP, RP-120382, Renesas Mobile Europe Ltd., "New WI: Improved minimum performance requirements for E-UTRA: Interference rejection," March 2012.

[8] X. Wang and H.V. Poor, "Iterative (Turbo) soft interference cancellation and decoding for coded CDMA," IEEE Trans. Commun., vol.47, pp.1046-1061, July 1999.

[9] T. Abe and T. Matsumoto, "Space-time turbo equalization in frequency selective MIMO channels," IEEE Trans. Veh. Technol., vol.52, no.3, pp.469-475, May 2003.

[10] 3GPP, R1-111562, Renesas Mobile Europe Ltd., "Interference aware receiver modeling at system level," May 2011.

[11] Y. Ohwatari, N. Miki, T. Asai, T. Abe, and H. Taoka, "Performance of advanced receiver employing interference rejection combining to suppress inter-cell interference in LTE-Advanced downlink," Proc. VTC2011-Fall, Sept. 2011

[12] 3GPP, TS36.211 (V10.2.0), "Physical channels and modulation," June 2011

[13] S.M. Alamouti, "A simple transmit diversity technique for wireless communications," IEEE J. Sel. Areas Commun., vol.16, no.8, pp.1451-1458, Oct. 1998.

[14] 3GPP, R4-121552, NTT DOCOMO, "Test coverage for improved minimum performance requirements for E-UTRA," March 2012.

[15] A.F. Naguib, N. Seshadri, and A.R. Calderbank, "Applications of space-time block codes and interference suppression for high capacity and high data rate wireless systems," Proc. Asilomar Conf. Computers, Signals and Systems, pp.1803-1810, Nov. 1998.

[16] P. Hoeher, S. Kaiser, and P. Robertson, "Two-dimensional pilotsymbol-aided channel estimation by Wiener filtering," Proc. ICASSP'97, pp.1845-1848, April 1997.

[17] J. Lee, R. Arnott, K. Hamabe, and N. Takano, "Adaptive modulation switching level control in high speed downlink packet access transmission," Proc. 3G Mobile Communication Technologies 2002, pp.156-159, May 2002.

[18] 3GPP, TS36.101 (V10.4.0), "User equipment (UE) radio transmission and reception," Oct. 2011

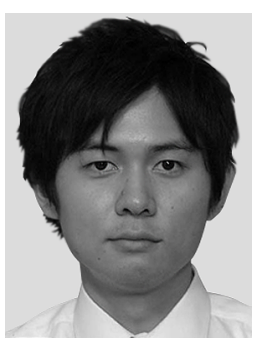

Yousuke Sano received his B.E. degree from The University of Electro-Communications, Tokyo, Japan in 2008, and M.E. degree from Tokyo Institute of Technology, Tokyo, Japan in 2010. Since 2010, he has been with the Research Laboratories, NTT DOCOMO, INC., Kanagawa Japan. His current interest includes wireless communication technologies.

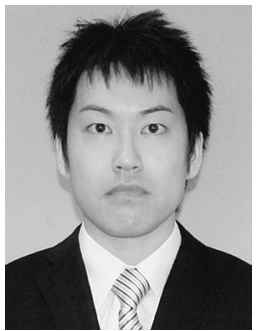

Yusuke Ohwatari received his B.E. degree in electronics engineering and M.E. degree in information science and technology in 2005 and 2007, respectively, both from Hokkaido University, Hokkaido, Japan. Since 2007, he has been with the Research Laboratories, NTT DOCOMO, INC., Kanagawa, Japan. His current interest includes multiple-input and multipleoutput (MIMO) systems for mobile communication systems. Mr. Ohwatari received the IEEE VTS Japan Chapter Young Researcher's Encouragement Award in 2006, the Best Paper Award from IEICE Japan in 2007, and the Young Researchers' Award of IEICE Japan in 2012.

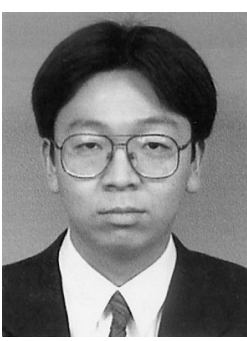

Nobuhiko Miki received his B.E. and M.E. degrees from Kyoto University, Kyoto, Japan in 1996 and 1998, respectively, and received his Dr. Eng. degree from Keio University, Yokohama, Japan in 2009. In 1998, he joined NTT Mobile Communications Network, Inc. (now NTT DOCOMO, INC.) His research interests include mobile communication systems. 


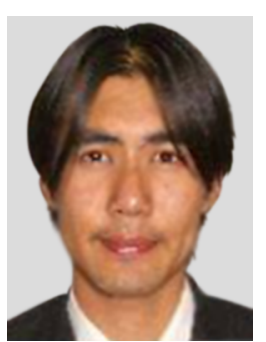

Yuta Sagae received his B.E. and M.E. degrees from Tokyo institute of technology, Japan in 2003 and 2005, respectively. In 2005, he joined NTT DOCOMO, INC. His research interests include mobile communication systems.

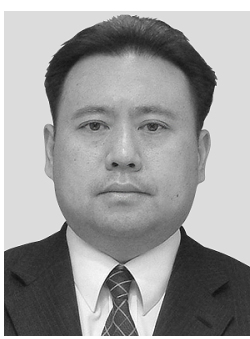

Yukihiko Okumura received B.S. and M.S. degrees in electrical engineering from the Tokyo University of Science, Tokyo, Japan, in 1989 and 1991, respectively, and his Ph.D. degree in engineering from Tohoku University, Sendai, Japan, in 2006. In 1991, He joined NTT Radio Communications Systems Laboratories of Nippon Telegraph and Telephone Corporation, and since 1992, he has been engaged in the research and development of wideband/broadband mobile radio communication technologies, terminals and systems, at NTT Mobile Communications Network, Inc. (now NTT DOCOMO, INC.). He is a senior member of IEEE.

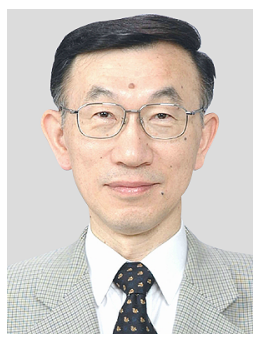

Yasutaka Ogawa received the B.E., M.E. and Ph.D. degrees from Hokkaido University, Sapporo, Japan, in 1973, 1975, and 1978, respectively. Since 1979, he has been with Hokkaido University, where he is currently a Professor of the Graduate School of Information Science and Technology. During 1992-1993, he was with ElectroScience Laboratory, the Ohio State University, U.S.A., as a Visiting Scholar, on leave from Hokkaido University. His interests are in adaptive antennas, mobile communications, super-resolution techniques, and MIMO systems. Dr. Ogawa received the Yasujiro Niwa outstanding paper award in 1978, the Young Researchers' Award of IEICE Japan in 1982, the Best Paper Award from IEICE Japan in 2007, and the Best Magazine Paper Award in 2011 from IEICE Communications Society. Dr. Ogawa is a Fellow of the IEEE.

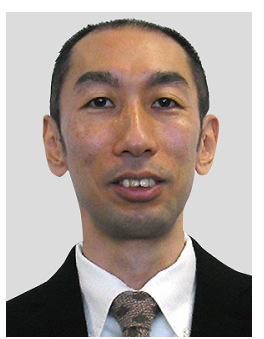

Takeo Ohgane received the B.E., M.E., and $\mathrm{Ph} . \mathrm{D}$. degrees in electronics engineering from Hokkaido University, Sapporo, Japan, in 1984, 1986, and 1994, respectively. From 1986 to 1992, he was with Communications Research Laboratory, Ministry of Posts and Telecommunications. From 1992 to 1995, he was on assignment at ATR Optical and Radio Communications Research Laboratory. Since 1995, he has been with Hokkaido University, where he is an Associate Professor. During 2005-2006, he was at Centre for Communications Research, University of Bristol, UK, as a Visiting Fellow. His interests are in MIMO signal processing for wireless communications. Dr. Ohgane received the IEEE AP-S Tokyo Chapter Young Engineer Award in 1993, the Young Researchers' Award of IEICE Japan in 1990, the Best Paper Award from IEICE Japan in 2007, and the Best Magazine Paper Award in 2011 from IEICE Communications Society. Dr. Ohgane is a member of the IEEE.

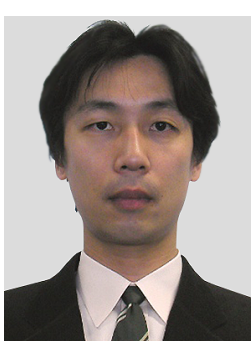

Toshihiko Nishimura received the B.S. and M.S. degrees in physics and Ph.D. degree in electronics engineering from Hokkaido University, Sapporo, Japan, in 1992, 1994, and 1997 , respectively. In 1998, he joined the Graduate School of Engineering (reorganized to Graduate School of Information Science and Technology at present) at Hokkaido University, where he is currently an Assistant Professor of the Graduate School of Information Science and Technology. His current research interests are in MIMO systems using smart antenna techniques. Dr. Nishimura received the Young Researchers' Award of IEICE Japan in 2000, the Best Paper Award from IEICE Japan in 2007, and the Best Magazine Paper Award in 2011 from IEICE Communications Society. Dr. Nishimura is a member of the IEEE. 